\section{Lyapunov-Based Tracking Control in the Presence of Uncertain Nonlinear Parameterizable Friction}

\author{
C. Makkar, G. Hu, W. G. Sawyer, and W. E. Dixon
}

\begin{abstract}
Modeling and compensation for friction effects has been a topic of considerable mainstream interest in motion control research. This interest is spawned from the fact that modeling nonlinear friction effects is a theoretically challenging problem, and compensating for the effects of friction in a controller has practical ramifications. If the friction effects in the system can be accurately modeled, there is an improved potential to design controllers that can cancel the effects; whereas, excessive steady-state tracking errors, oscillations, and limit cycles can result from controllers that do not accurately compensate for friction. A tracking controller is developed in this paper for a general Euler-Lagrange system that contains a new continuously differentiable friction model with uncertain nonlinear parameterizable terms. To achieve the semi-global asymptotic tracking result, a recently developed integral feedback compensation strategy is used to identify the friction effects online, assuming exact model knowledge of the remaining dynamics. A Lyapunov-based stability analysis is provided to conclude the tracking and friction identification results. Experimental results illustrate the tracking and friction identification performance of the developed controller.
\end{abstract}

Index Terms_Friction, Lyapunov methods, nonlinear systems, uncertain systems.

\section{INTRODUCTION}

The modeling and compensation for friction effects has been a topic of considerable mainstream interest in motion control research. This interest is spawned from the fact that modeling nonlinear friction effects is a theoretically challenging problem, and compensating for the effects of friction in a controller has practical ramifications. If the friction effects in a system can be accurately modeled, there is an improved potential to design controllers that can cancel the effects (e.g., model-based controllers); whereas, excessive steady-state tracking errors, oscillations, and limit cycles can result from controllers that do not accurately compensate for friction. Friction is exaggerated at low velocities, which are present in high-precision and high-performance motion control systems; unfortunately, a general model for friction which describes the effects at low velocity has not been universally accepted. Many models of friction have been proposed to deal with the various regimes of friction, each with their own merits and limitations. See [1], [3], [9], [11]-[13], [16], [30], and [33] for a survey of friction modeling and control results. Given the difficulty in accurately modeling and compensating for friction effects, researchers have proposed a variety of (typically offline) friction estimation schemes with the objective of identifying the friction effects. For example, in [8], an offline maximum likelihood, frequency-based approach (differential binary excitation) is proposed to estimate Coulomb friction effects. Another frequency-based offline friction identification approach was

Manuscript received October 26, 2005; revised May 23, 2006 and January 17, 2007. Recommended by Associate Editor M.-Q. Xiao. This research was supported by the AFOSR under Contract F49620-03-1-0381 and by the National Science Foundation CAREER award with project number (CMS) 0547448 at the University of Florida.

The authors are with the Department of Mechanical and Aerospace Engineering, University of Florida, Gainesville, FL 32611-6250 USA (e-mail: cmakkar@ufl.edu; gqhu@ufl.edu; wgsawyer@ufl.edu; wdixon@uff.edu).

Color versions of one or more of the figures in this article are available at http://ieeexplore.ieee.org.

Digital Object Identifier 10.1109/TAC.2007.904254 proposed in [19]. Specifically, the approach in [19] uses a kind of frequency-domain linear regression model derived from Fourier analysis of the periodic steady-state oscillations of the system. The approach in [19] requires a periodic excitation input with sufficiently large amplitude and/or frequency content. A new offline friction identification tool is proposed in [20] where the static-friction models are not required to be linear parameterizable. However the offline optimization result in [20] is limited to single degree-of-freedom systems where the initial and final velocities are equal. Another frequency domain identification strategy developed to identify dynamic model parameters for presliding behavior is given in [14]. Additional identification methods include least-squares [5] and Kalman filtering [15].

In addition to friction identification schemes, researchers have developed adaptive, robust, and learning controllers to achieve a control objective while accommodating for the friction effects, but not necessarily identifying friction. For example, given a desired trajectory that is periodic and not constant over some interval of time, the development in [9] provides a learning control approach to damp out periodic steady-state oscillations due to friction. As stated in [9], a periodic signal is applied to the system and when the system reaches a steady-state oscillation, the learning update law is applied. In [22], a discontinuous linearizing controller was proposed along with an adaptive estimator to achieve an exponentially stable tracking result that estimates the unknown Coulomb friction coefficient. However, [35] describes a technical error in the result presented in [22] that invalidates the result. Additional development is provided in [35] that modifies the result in [22] to achieve asymptotic Coulomb friction coefficient estimation provided a persistence of excitation condition is satisfied. In [31], Tomei proposed a robust adaptive controller where only instantaneous friction is taken into account (dynamic friction effects are not included).

Motivated by the desire to include dynamic friction models in the control design, numerous researchers have embraced the LuGre friction model proposed in [7]. For example, the result in [31] was extended in [32] to include the LuGre friction model [7], resulting in an asymptotic tracking result for square integrable disturbances. Robust adaptive controllers were also proposed in [17] and [29] to account for the LuGre model. Canudas et al. investigated the development of observer-based approaches for the LuGre model in [7]. In [4], Canudas and Lichinsky proposed an adaptive friction compensation method, and in [6] Canudas and Kelly proposed a passivity-based friction compensation term to achieve global asymptotic tracking using the LuGre model. In [2], Barabanov and Ortega developed necessary and sufficient conditions for the passivity of the LuGre model. In [33], three observer-based control schemes were proposed assuming exact model knowledge of the system dynamics. The results in [33] were later extended to include two adaptive observers to account for selected uncertainty in the model. The observer-based design in [33] was further extended in [12]. Specifically, in [12], a partial-state feedback exact model knowledge controller was developed to achieve global exponential link position tracking of a robot manipulator. Two adaptive, partial-state feedback global asymptotic controllers were also proposed in [12] that compensate for selected uncertainty in the system model. In addition, a new adaptive control technique was proposed in [12] to compensate for the nonlinear parameterizable Stribeck effect, where the average square integral of the position tracking errors were forced to an arbitrarily small value.

In this paper and in the preliminary results in [23], a tracking controller is developed for a general Euler-Lagrange system that contains a new continuously differentiable friction model with uncertain nonlinear parameterizable terms. Friction models are often based on the as- 
sumption that the friction coefficient is constant with sliding speed and have a singularity at the onset of slip. Such models typically include a signum function of the velocity to assign the direction of friction force (e.g., [21], [30]), and many other models are only piecewise continuous (e.g., the LuGre model in [7]). In [24], we proposed a new friction model that captures a number of essential aspects of friction without involving discontinuous or piecewise continuous functions. The simple continuously differentiable model represents a foundation that captures the major effects reported and discussed in friction modeling and experimentation and the model is generic enough that other subtleties such as frictional anisotropy with sliding direction can be addressed by mathematically distorting this model without compromising the continuous differentiability. Based on the fact that the model is continuously differentiable, a new integral feedback compensation term originally proposed in [34] is exploited to enable a semi-global tracking result while identifying the friction on-line, assuming exact model knowledge of the remaining dynamics. A Lyapunov-based stability analysis is provided to conclude the tracking and friction identification results. Experimental results show two orders of magnitude improvement in tracking control over a proportional derivative (PD) controller, and a one order of magnitude improvement over the model-based controller. Experimental results are also used to illustrate that the experimentally identified friction can be approximated by the model in [24].

\section{DYNAMIC MODEL AND PROPERTIES}

The class of nonlinear dynamic systems considered in this paper are assumed to be modeled by the following general Euler-Lagrange formulation:

$$
M(q) \ddot{q}+V_{m}(q, \dot{q}) \dot{q}+G(q)+f(\dot{q})=\tau(t) .
$$

In (1), $M(q) \in \mathbb{R}^{n \times n}$ denotes the inertia matrix, $V_{m}(q, \dot{q}) \in \mathbb{R}^{n \times n}$ denotes the centripetal-Coriolis matrix, $G(q) \in \mathbb{R}^{n}$ denotes the gravity vector, $f(\dot{q}) \in \mathbb{R}^{n}$ denotes a friction vector, $\tau(t) \in \mathbb{R}^{n}$ represents the torque input control vector, and $q(t), \dot{q}(t), \ddot{q}(t) \in \mathbb{R}^{n}$ denote the link position, velocity, and acceleration vectors, respectively. The friction term $f(\dot{q})$ in (1) is assumed to have the following form as in [24]:

$$
f(\dot{q})=\gamma_{1}\left(\tanh \left(\gamma_{2} \dot{q}\right)-\tanh \left(\gamma_{3} \dot{q}\right)\right)+\gamma_{4} \tanh \left(\gamma_{5} \dot{q}\right)+\gamma_{6} \dot{q}
$$

where $\gamma_{i} \in \mathbb{R} \forall i=1,2, \ldots 6$ denote unknown positive constants. The friction model in (2) has the following properties: 1) it is symmetric about the origin, 2) it has a static coefficient of friction, 3) it exhibits the Stribeck effect where the friction coefficient decreases from the static coefficient of friction with increasing slip velocity near the origin, 4) it includes a viscous dissipation term, and 5) it has a Coulombic friction coefficient in the absence of viscous dissipation. To a good approximation, the static friction coefficient is given by $\gamma_{1}+\gamma_{4}$, and the Stribeck effect is captured by $\tanh \left(\gamma_{2} \dot{q}\right)-\tanh \left(\gamma_{3} \dot{q}\right)$. The Coulombic friction coefficient is given by $\gamma_{4} \tanh \left(\gamma_{5} \dot{q}\right)$, and the viscous dissipation is given by $\gamma_{6} \dot{q}$. For further details regarding the friction model, see [24].

The subsequent development is based on the assumption that $q(t)$ and $\dot{q}(t)$ are measurable and that $M(q), V_{m}(q, \dot{q}), G(q)$ are known. Moreover, the following properties and assumptions will be exploited in the subsequent development:

Property 1: The inertia matrix $M(q)$ is symmetric, positive definite, and satisfies the following inequality $\forall y(t) \in \mathbb{R}^{n}$ :

$$
m_{1}\|y\|^{2} \leq y^{T} M(q) y \leq \bar{m}(\|y\|)\|y\|^{2}
$$

where $m_{1} \in \mathbb{R}$ is a known positive constant, $\bar{m}(y) \in \mathbb{R}$ is a known positive function, and $\|\cdot\|$ denotes the standard Euclidean norm.

Property 2: If $q(t) \in \mathcal{L}_{\infty}$, then $\partial M(q) / \partial q$, and $\partial^{2} M(q) / \partial q^{2}$ exist and are bounded. Moreover, if $q(t), \dot{q}(t) \in \mathcal{L}_{\infty}$ then $V_{m}(q, \dot{q})$ and $G(q)$ are bounded.

Property 3: Based on the structure of $f(\dot{q})$ given in $(2), f(\dot{q}), \dot{f}(\dot{q})$, and $\ddot{f}(\dot{q})$ exist and are bounded provided $q(t), \dot{q}(t), \ddot{q}(t), \dddot{q}(t) \in \mathcal{L}_{\infty}$.

\section{ERROR SYSTEM DEVELOPMENT}

The control objective is to ensure that the system tracks a desired trajectory, denoted by $q_{d}(t)$, that is assumed to be designed such that $q_{d}(t), \dot{q}_{d}(t), \ddot{q}_{d}(t), \dddot{q}_{d}(t) \in \mathbb{R}^{n}$ exist and are bounded. A position tracking error, denoted by $e_{1}(t) \in \mathbb{R}^{n}$, is defined as follows to quantify the control objective:

$$
e_{1} \triangleq q_{d}-q
$$

The following filtered tracking errors, denoted by $e_{2}(t), r(t) \in \mathbb{R}^{n}$, are defined to facilitate the subsequent design and analysis:

$$
\begin{array}{r}
e_{2} \triangleq \dot{e}_{1}+\alpha_{1} e_{1} \\
r \triangleq \dot{e}_{2}+\alpha_{2} e_{2}
\end{array}
$$

where $\alpha_{1}, \alpha_{2} \in \mathbb{R}$ denote positive constants. The filtered tracking error $r(t)$ is not measurable since the expression in (6) depends on $\ddot{q}(t)$.

After premultiplying (6) by $M(q)$, the following expression can be obtained:

$$
\begin{aligned}
M(q) r=M(q) \ddot{q}_{d}+V_{m}(q, \dot{q}) \dot{q}+ & G(q)+f(\dot{q})-\tau(t) \\
& +M(q) \alpha_{1} \dot{e}_{1}+M(q) \alpha_{2} e_{2}
\end{aligned}
$$

where (1), (4), and (5) were utilized. Based on the expression in (7) the control torque input is designed as follows:

$$
\begin{aligned}
\tau(t)=M(q) \ddot{q}_{d}+V_{m}(q, \dot{q}) \dot{q}+G(q)+ & M(q) \alpha_{1} \dot{e}_{1} \\
& +M(q) \alpha_{2} e_{2}+\mu(t)
\end{aligned}
$$

where $\mu(t) \in \mathbb{R}^{n}$ denotes a subsequently designed control term. By substituting (8) into (7), the following expression can be obtained:

$$
M(q) r=f(\dot{q})-\mu(t) .
$$

From (9), it is evident that if $r(t) \rightarrow 0$, then $\mu(t)$ will identify the friction dynamics; therefore, the objective is to design the control term $\mu(t)$ to ensure that $r(t) \rightarrow 0$. To facilitate the design of $\mu(t)$, we differentiate (9) as follows:

$$
M(q) \dot{r}=\dot{f}(\dot{q})-\dot{\mu}(t)-\dot{M}(q) r .
$$

Based on (10) and the subsequent stability analysis, $\mu(t)$ is designed as follows ${ }^{1}$ :

$$
\begin{aligned}
\mu(t)=\left(k_{s}+1\right) e_{2}(t)-\left(k_{s}+1\right) e_{2}(0) & \\
& +\int_{0}^{t}\left[\left(k_{s}+1\right) \alpha_{2} e_{2}(\tau)+\beta \operatorname{sgn}\left(e_{2}(\tau)\right)\right] d \tau
\end{aligned}
$$

${ }^{1}$ The expression in (11) for $\mu(t)$ does not depend on the unmeasurable filtered tracking error term $r(t)$. However, the time derivative of $\mu(t)$ (which is not implemented) can be expressed as a function of $r(t)$ 
where $k_{s} \in \mathbb{R}$ and $\beta \in \mathbb{R}$ are positive constants. The time derivative of (11) is given as ${ }^{2}$

$$
\dot{\mu}(t)=\left(k_{s}+1\right) r+\beta \operatorname{sgn}\left(e_{2}\right) .
$$

After substituting (12) into (10), the following closed-loop error system can be obtained:

$$
M(q) \dot{r}=-\frac{1}{2} \dot{M}(q) r-\left(k_{s}+1\right) r-e_{2}-\beta \operatorname{sgn}\left(e_{2}\right)+N(t)
$$

where $N(q, \dot{q}, t) \in \mathbb{R}^{n}$ denotes the following unmeasurable auxiliary term:

$$
N(q, \dot{q}, t) \triangleq \dot{f}(\dot{q})-\frac{1}{2} \dot{M}(q) r+e_{2} .
$$

To facilitate the subsequent analysis, another unmeasurable auxiliary term $N_{d}(t) \in \mathbb{R}^{n}$ is defined as follows:

$$
\begin{aligned}
N_{d}(t) \triangleq & \frac{\partial f\left(\dot{q}_{d}\right)}{\partial \dot{q}_{d}} \ddot{q}_{d} \\
= & \gamma_{1} \gamma_{2} \ddot{q}_{d}-\gamma_{1} \gamma_{2} \ddot{q}_{d}\left\|\tanh \left(\gamma_{2} \dot{q}_{d}\right)\right\|^{2} \\
& -\gamma_{1} \gamma_{3} \ddot{q}_{d}+\gamma_{1} \gamma_{3} \ddot{q}_{d}\left\|\tanh \left(\gamma_{3} \dot{q}_{d}\right)\right\|^{2}+\gamma_{4} \gamma_{5} \ddot{q}_{d} \\
& -\gamma_{4} \gamma_{5} \ddot{q}_{d}\left\|\tanh \left(\gamma_{5} \dot{q}_{d}\right)\right\|^{2}+\gamma_{6} \ddot{q}_{d} .
\end{aligned}
$$

The time derivative of (14) is given as follows:

$$
\begin{aligned}
\dot{N}_{d}(t)= & \frac{\partial^{2} f\left(\dot{q}_{d}\right)}{\partial \dot{q}_{d}^{2}} \ddot{q}_{d}^{2}+\frac{\partial f\left(\dot{q}_{d}\right)}{\partial \dot{q}_{d}} \dddot{q}_{d} \\
= & \dddot{q}_{d}\left(\gamma_{1} \gamma_{2}-\gamma_{1} \gamma_{3}+\gamma_{4} \gamma_{5}+\gamma_{6}\right) \\
& -\gamma_{1} \gamma_{2} \ddot{q}_{d}\left\|\tanh \left(\gamma_{2} \dot{q}_{d}\right)\right\|^{2} \\
& +\gamma_{1} \gamma_{3} \dddot{q}_{d}\left\|\tanh \left(\gamma_{3} \dot{q}_{d}\right)\right\|^{2}-\gamma_{4} \gamma_{5} \dddot{q}_{d}\left\|\tanh \left(\gamma_{5} \dot{q}_{d}\right)\right\|^{2} \\
& -2 \gamma_{1} \gamma_{2}^{2}\left\|\ddot{q}_{d}\right\|^{2} \tanh \left(\gamma_{2} \dot{q}_{d}\right)\left[1-\left\|\tanh \left(\gamma_{2} \dot{q}_{d}\right)\right\|^{2}\right] \\
& +2 \gamma_{1} \gamma_{3}^{2}\left\|\ddot{q}_{d}\right\|^{2} \tanh \left(\gamma_{3} \dot{q}_{d}\right)\left[1-\left\|\tanh \left(\gamma_{3} \dot{q}_{d}\right)\right\|^{2}\right] \\
& -2 \gamma_{4} \gamma_{5}^{2}\left\|\ddot{q}_{d}\right\|^{2} \tanh \left(\gamma_{5} \dot{q}_{d}\right) \\
& \times\left[1-\left\|\tanh \left(\gamma_{5} \dot{q}_{d}\right)\right\|^{2}\right] .
\end{aligned}
$$

After adding and subtracting (14), the closed-loop error system in (13) can be expressed as follows:

$M(q) \dot{r}=-\frac{1}{2} \dot{M}(q) r-\left(k_{s}+1\right) r-e_{2}-\beta \operatorname{sgn}\left(e_{2}\right)+\tilde{N}(t)+N_{d}(t)$

where the unmeasurable auxiliary term $\tilde{N}(t) \in \mathbb{R}^{n}$ is defined as

$$
\tilde{N}(t) \triangleq N(t)-N_{d}(t)
$$

Based on the expressions in (14) and (15), the following inequalities can be developed:

$$
\begin{aligned}
\left\|N_{d}(t)\right\| \leq\left\|\ddot{q}_{d}\right\| \cdot\left|\gamma_{1} \gamma_{2}+\gamma_{4} \gamma_{5}+\gamma_{6}-\gamma_{1} \gamma_{3}\right| \leq \zeta_{N_{d}} \\
\left\|\dot{N}_{d}(t)\right\| \leq\left\|\ddot{q}{ }_{d}\right\| \cdot\left|\gamma_{1} \gamma_{2}+\gamma_{4} \gamma_{5}+\gamma_{6}-\gamma_{1} \gamma_{3}\right| \\
\quad+\left\|\ddot{q}_{d}\right\|^{2}\left(2 \gamma_{1} \gamma_{2}^{2}+2 \gamma_{1} \gamma_{3}^{2}+2 \gamma_{4} \gamma_{5}^{2}\right) \leq \zeta_{N_{d}} 2
\end{aligned}
$$

where $\zeta_{N_{d}}, \zeta_{N_{d} 2} \in \mathbb{R}$ are known positive constants.

\section{STABILITY ANALYSIS}

Theorem 1: The controller given in (8) and (11) ensures that the position tracking error is regulated in the sense that

$$
e_{1}(t) \rightarrow 0 \text { as } t \rightarrow \infty
$$

provided $\beta$ is selected according to the following sufficient condition:

$$
\beta>\zeta_{N_{d}}+\frac{1}{\alpha_{2}} \zeta_{N_{d}}
$$

where $\zeta_{N_{d}}$ and $\zeta_{N_{d}}$ are introduced in (18) and (19), respectively, and $k_{s}$ is selected sufficiently large to yield a semi-global asymptotic result. The control system represented by (8) and (11) also ensures that all system signals are bounded under closed-loop operation and that the friction in the system can be identified in the sense that

$$
f(\dot{q})-\mu(t) \rightarrow 0 \quad \text { as } \quad t \rightarrow \infty .
$$

Proof: Let $\mathcal{D} \subset \mathbb{R}^{3 n+1}$ be a domain containing $y(t)=0$, where $y(t) \in \mathbb{R}^{3 n+1}$ is defined as $y(t) \triangleq\left[z^{T}(t) \sqrt{P(t)}^{T}\right.$ where $z(t) \in \mathbb{R}^{3 n}$ is defined as $z(t) \triangleq\left[\begin{array}{lll}e_{1}^{T} & e_{2}^{T} & r^{T}\end{array}\right]^{T}$, and the auxiliary function $P(t) \in \mathbb{R}$ is defined as

$$
P(t) \triangleq \beta\left\|e_{2}(0)\right\|-e_{2}(0)^{T} N_{d}(0)-\int_{0}^{t} L(\tau) d \tau
$$

where $\beta \in \mathbb{R}$ is nonnegative by design.

In (21), the auxiliary function $L(t) \in \mathbb{R}$ is defined as

$$
L(t) \triangleq r^{T}\left(N_{d}(t)-\beta \operatorname{sgn}\left(e_{2}\right)\right) .
$$

The derivative $\dot{P}(t) \in \mathbb{R}$ can be expressed as

$$
\dot{P}(t)=-L(t)=-r^{T}\left(N_{d}(t)-\beta_{\operatorname{sgn}}\left(e_{2}\right)\right) .
$$

Provided the sufficient condition introduced in (20) is satisfied, the following inequality can be obtained ${ }^{3}$ :

$$
\int_{0}^{t} L(\tau) d \tau \leq \beta\left|e_{2}(0)\right|-e_{2}(0)^{T} N_{d}(0) .
$$

Hence, (24) can be used to conclude that $P(t) \geq 0$. Let $V(y, t)$ : $\mathcal{D} \times[0, \infty) \rightarrow \mathbb{R}$ be a continuously differentiable positive-definite function defined as

$$
V(y, t) \triangleq e_{1}^{T} e_{1}+\frac{1}{2} e_{2}^{T} e_{2}+\frac{1}{2} r^{T} M(q) r+P
$$

that can be bounded as

$$
\lambda_{1}\|y\|^{2} \leq V(y, t) \leq \lambda_{2}(y)\|y\|^{2}
$$

provided the sufficient condition introduced in (20) is satisfied. In (26), $\lambda_{1}, \lambda_{2}(y) \in \mathbb{R}$ are defined as

$$
\lambda_{1} \triangleq \frac{1}{2} \min \left\{1, m_{1}\right\}, \quad \lambda_{2}(y) \triangleq \max \left\{\frac{1}{2} \bar{m}(y), 1\right\}
$$

${ }^{3}$ See $[25]$ for details. 
where $m_{1}, \bar{m}(q)$ are introduced in (3). After taking the time derivative of $(25), \dot{V}(y, t)$ can be expressed as

$$
\dot{V}=r^{T} M(q) \dot{r}+\frac{1}{2} r^{T} \dot{M}(q) r+e_{2}^{T} \dot{e}_{2}+2 e_{1}^{T} \dot{e}_{1}+\dot{P}
$$

After utilizing (5), (6), (16), and (23), $\dot{V}(y, t)$ can be simplified as follows:

$$
\dot{V}=r^{T} \tilde{N}(t)-\left(k_{s}+1\right)\|r\|^{2}-\alpha_{2}\left\|e_{2}\right\|^{2}-2 \alpha_{1}\left\|e_{1}\right\|^{2}+2 e_{2}^{T} e_{1} .
$$

Because $2 e_{2}^{T}(t) e_{1}(t)$ can be upper bounded as

$$
2 e_{2}^{T} e_{1} \leq\left\|e_{1}\right\|^{2}+\left\|e_{2}\right\|^{2}
$$

$\dot{V}(y, t)$ can be upper bounded using the squares of the components of $z(t)$ as follows:

$$
\dot{V} \leq r^{T} \tilde{N}-\left(k_{s}+1\right)\|r\|^{2}-\alpha_{2}\left\|e_{2}\right\|^{2}-2 \alpha_{1}\left\|e_{1}\right\|^{2}+\left\|e_{1}\right\|^{2}+\left\|e_{2}\right\|^{2} .
$$

By exploiting the mean value theorem, the following inequality can be developed for $(17)^{4}$ :

$$
\|\tilde{N}\| \leq \rho(\|z\|)\|z\|
$$

By exploiting the inequality in (28), the expression in (27) can be rewritten as

$$
\dot{V} \leq-\lambda_{3}\|z\|^{2}-\left(k_{s}\|r\|^{2}-\rho(\|z\|)\|r\|\|z\|\right)
$$

where $\lambda_{3} \triangleq \min \left\{2 \alpha_{1}-1, \alpha_{2}-1,1\right\}$ and the bounding function $\rho(\|z\|) \in \mathbb{R}$ is a positive globally invertible nondecreasing function; hence, $\alpha_{1}, \alpha_{2}$ must be chosen according to the following conditions:

$$
\alpha_{1}>\frac{1}{2}, \quad \alpha_{2}>1 \text {. }
$$

After completing the squares for the second and third term in (29), the following expression can be obtained:

$$
\dot{V} \leq-\lambda_{3}\|z\|^{2}+\frac{\rho^{2}(\|z\|)\|z\|^{2}}{4 k_{s}} .
$$

The following expression can then be obtained from (30):

$$
\dot{V} \leq-W(y)
$$

where $W(y)=c\|z\|^{2}$, for some positive constant $c \in \mathbb{R}$, is a continuous positive semi-definite function that is defined on the following domain:

$$
D \triangleq\left\{y \in \mathbb{R}^{3 n+1} \mid\|y\| \leq \rho^{-1}\left(2 \sqrt{\lambda_{3} k_{s}}\right)\right\} .
$$

The inequalities in (26) and (31) can be used to show that $V \in \mathcal{L}_{\infty}$ in $\mathcal{D}$; hence, $e_{1}, e_{2}$, and $r \in \mathcal{L}_{\infty}$ in $\mathcal{D}$. Given that $e_{1}, e_{2}$, and $r \in \mathcal{L}_{\infty}$ in $\mathcal{D}$, standard linear analysis methods (e.g., Lemma 1.4 of [10]) can be used to prove that $\dot{e}_{1}, \dot{e}_{2} \in \mathcal{L}_{\infty}$ in $\mathcal{D}$ from (5) and (6). Since $e_{1}$, $e_{2}, r \in \mathcal{L}_{\infty}$ in $\mathcal{D}$, the assumption that $q_{d}(t), \dot{q}_{d}(t), \ddot{q}_{d}(t)$ exist and are bounded can be used along with (4)-(6) to conclude that $q, \dot{q}, \ddot{q} \in \mathcal{L}_{\infty}$ in $\mathcal{D}$. Since $q, \dot{q} \in \mathcal{L}_{\infty}$ in $\mathcal{D}$, Property 2 can be used to conclude that $M(q), V_{m}(q, \dot{q}), G(q)$, and $f(\dot{q}) \in \mathcal{L}_{\infty}$ in $\mathcal{D}$. From (8) and (11), we can show that $\mu, \tau \in \mathcal{L}_{\infty}$ in $\mathcal{D}$. Given that $r \in \mathcal{L}_{\infty}$ in $\mathcal{D},(12)$ can be used to show that $\dot{\mu} \in \mathcal{L}_{\infty}$ in $\mathcal{D}$. Property 2 and Property 3 can be used to show that $\dot{f}(q)$ and $\dot{M}(q) \in \mathcal{L}_{\infty}$ in $\mathcal{D}$; hence, (10) can be used to show that $\dot{r} \in \mathcal{L}_{\infty}$ in $\mathcal{D}$. Given that $\dot{r} \in \mathcal{L}_{\infty}$ in $\mathcal{D}$, then (4)-(6) can

\footnotetext{
${ }^{4}$ See [25] and [34] for details
}

be used to conclude that $\dddot{q} \in \mathcal{L}_{\infty}$ in $\mathcal{D}$. Since $\dot{e}_{1}, \dot{e}_{2}, \dot{r} \in \mathcal{L}_{\infty}$ in $\mathcal{D}$, the definitions for $W(y)$ and $z(t)$ can be used to prove that $W(y)$ is uniformly continuous in $\mathcal{D}$.

Let $\mathcal{S} \subset \mathcal{D}$ denote a set defined as follows 5 :

$$
\mathcal{S} \triangleq\left\{y(t) \subset \mathcal{D} \mid \lambda_{2}(y)\|y\|^{2}<\lambda_{1}\left(\rho^{-1}\left(2 \sqrt{\lambda_{3} k_{s}}\right)\right)^{2}\right\} .
$$

Theorem 8.4 of [18] can now be invoked to state that

$$
c\|z(t)\|^{2} \rightarrow 0 \quad \text { as } \quad t \rightarrow \infty \quad \forall y(0) \in \mathcal{S} .
$$

Remark 1: The expressions in (5), (13), (21), and (22) can be written as

$$
\begin{aligned}
\dot{e}_{1} & =e_{2}-\alpha_{1} e_{1} \\
\dot{e}_{2} & =r-\alpha_{2} e_{2} \\
M \dot{r} & =-\frac{1}{2} \dot{M} r-\left(k_{s}+1\right) r-e_{2}-\beta \operatorname{sgn}\left(e_{2}\right)+\tilde{N}+N_{d} \\
\dot{P} & =-L=-r^{T}\left(N_{d}-\beta \operatorname{sgn}\left(e_{2}\right)\right) .
\end{aligned}
$$

From (34)-(37), it can be seen that the differential equations describing the closed-loop system for which the stability analysis is being performed has a discontinuous right-hand side. Let $y=\left[\begin{array}{lll}e_{1}^{T} & e_{2}^{T} & r^{T}\end{array} P^{T}\right]^{T}$ and $f(y, t): \mathbb{R}^{3 n+1}$ denote the right-hand side of (34)-(37). For $W(y)$ to be uniformly continuous for $y(0) \in \mathcal{S}$, it is required that a solution exists for $\dot{y}=f(y, t)$; it is important to comment on the existence of solutions to (34)-(37). To this end, the arguments used in [27] and [28] can be used to discuss the existence of Filippov's generalized solution to (34)-(37). See remark 4 of [34] for details.

Based on the definition of $z(t),(33)$ can be used to show that

$$
r(t) \rightarrow 0 \quad \text { as } \quad t \rightarrow \infty \quad \forall y(0) \in \mathcal{S}
$$

Hence, from (5) and (6), standard linear analysis methods (e.g., Lemma 1.6 of [10]) can be used to prove that

$$
e_{1}(t) \rightarrow 0 \quad \text { as } \quad t \rightarrow \infty \quad \forall y(0) \in \mathcal{S} .
$$

The result in (38) can also be used to conclude from (9) that

$$
\mu(t)-f(\dot{q}) \rightarrow 0 \quad \text { as } \quad t \rightarrow \infty \quad \forall y(0) \in \mathcal{S} .
$$

\section{EXPERIMENTAL RESULTS}

To illustrate the performance of the controller, a testbed was constructed consisting of a circular disk made of aluminium mounted on a NSK direct-drive switched reluctance motor. A rectangular Nylon block was mounted on a pneumatic linear thruster to apply an external friction load to the rotating disk. A pneumatic regulator maintained a constant pressure of 15 pounds per square inch on the circular disk. Data acquisition and control implementation were performed at a frequency of $1.0 \mathrm{kHz}$ using the ServoToGo I/O board.

The dynamics for the testbed are given as

$$
\tau(t)=\underbrace{\left[I_{m}+0.5 m a^{2}\right][\ddot{q}]}_{M(q) \ddot{q}}+f(\dot{q})
$$

where $I_{m}$ (rotor moment of inertia) $=0.255 \mathrm{~kg}-\mathrm{m}^{2}, m$ (mass of the circular disk $)=3.175 \mathrm{~kg}, a$ (radius of the disk $)=0.25527 \mathrm{~m}$, and the friction torque $f(\dot{q}) \in \mathbb{R}$ is defined in (2). The control torque input

${ }^{5}$ The region of attraction in (32) can be made arbitrarily large to include any initial conditions by increasing the control gain $k_{s}$ (i.e., a semi-global type of stability result) [34]. 


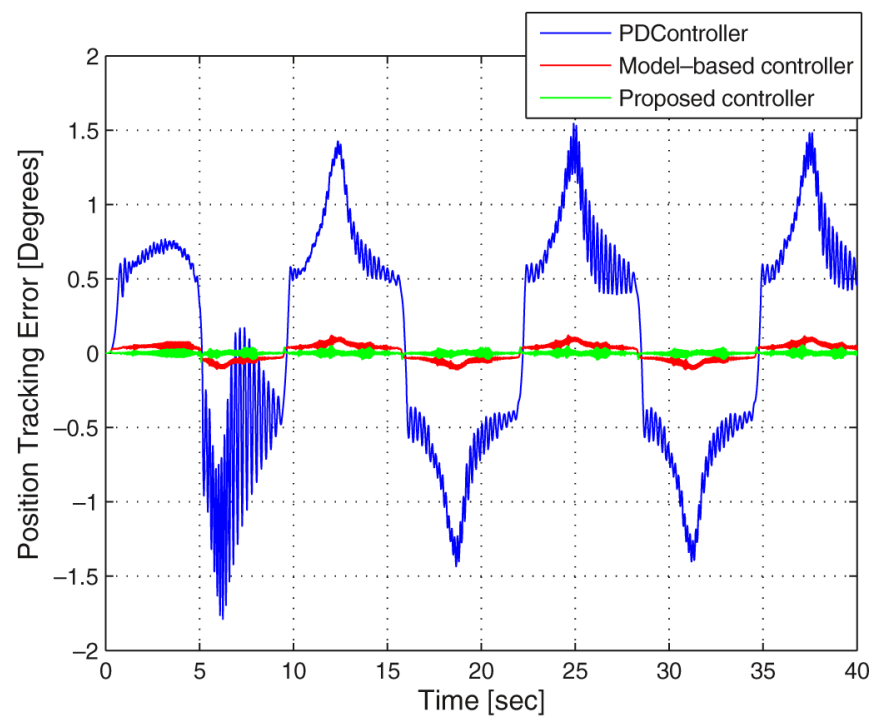

Fig. 1. Comparison of position tracking errors.

$\tau(t)$ given in (8) is simplified (i.e., the centripetal-Coriolis matrix and gravity terms do not exist in this testbed) as

$$
\tau(t)=M(q) \ddot{q}_{d}+M(q) \alpha_{1} \dot{e}_{1}+M(q) \alpha_{2} e_{2}+\mu(t)
$$

where $\mu(t)$ is the adaptive friction identification term defined in (11). The desired disk trajectory was selected similar to the one used in [12] to emphasize a low-speed direction transition as follows (in degrees):

$$
q_{d}(t)=11.25 \tan ^{-1}(3.0 \sin (0.5 t))\left(1-\exp \left(-0.01 t^{3}\right)\right) .
$$

For all experiments, the rotor velocity signal is obtained by applying a standard backwards difference algorithm to the position signal. All states were initialized to zero. In addition, the integral structure of the adaptive term in (40) was computed on-line via a standard trapezoidal algorithm.

\section{A. Experiment 1}

In the first experiment, no external load from the thruster was applied to the circular disk. In addition to the controller given in (39) and (40), a PD controller and a model-based controller were also implemented for comparison. The PD controller was implemented as:

$$
\tau(t)=k_{d} \dot{e}_{1}+k_{p} e_{1}
$$

where $k_{d} \in \mathbb{R}$ is the derivative gain and $k_{p} \in \mathbb{R}$ is the proportional gain. The model-based controller was implemented with standard friction feedforward terms as:

$$
\tau(t)=M(q) \ddot{q}_{d}+M(q) \alpha_{1} \dot{e}_{1}+M(q) \alpha_{2} e_{2}+k_{c} \operatorname{sgn}(\dot{q})+k_{v} \dot{q}+k_{s} q
$$

where $k_{c} \in \mathbb{R}$ is the Coulomb friction coefficient, $k_{v} \in \mathbb{R}$ is the viscous friction coefficient, and $k_{s} \in \mathbb{R}$ is the static friction coefficient.

A comparison of the position tracking error from each controller is seen in Figs. 1 and 2. The friction identification term in (11) from the proposed controller obtained from the experiment is given in Fig. 3.

\section{B. Experiment 2}

An external friction load was induced on the system. An external moment load of $12.774 \mathrm{Nm}$ was applied to the circular disk using the linear thruster. The desired disk trajectory of (41) was again utilized.

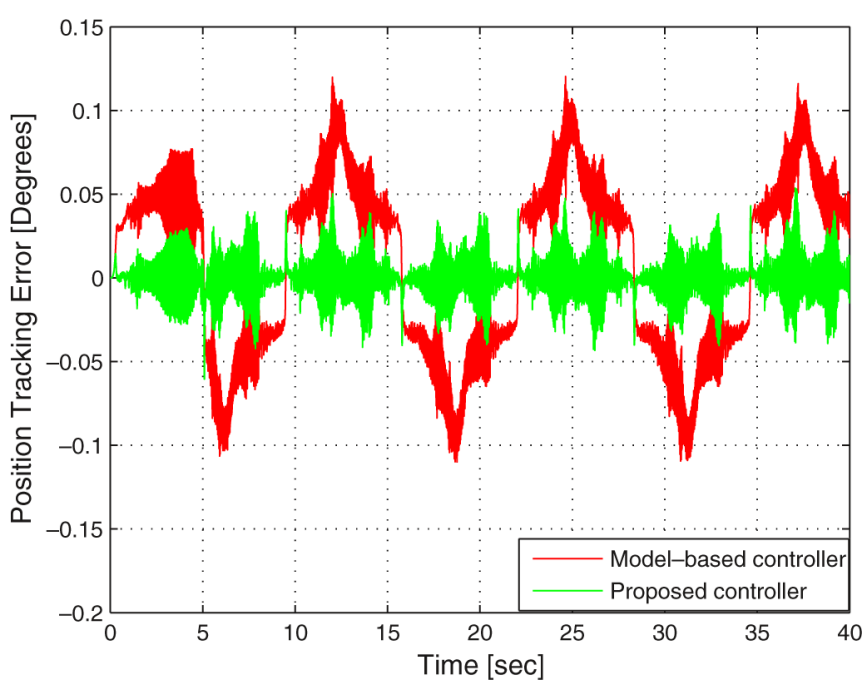

Fig. 2. Comparison of position tracking errors from the model-based controller and the proposed controller.

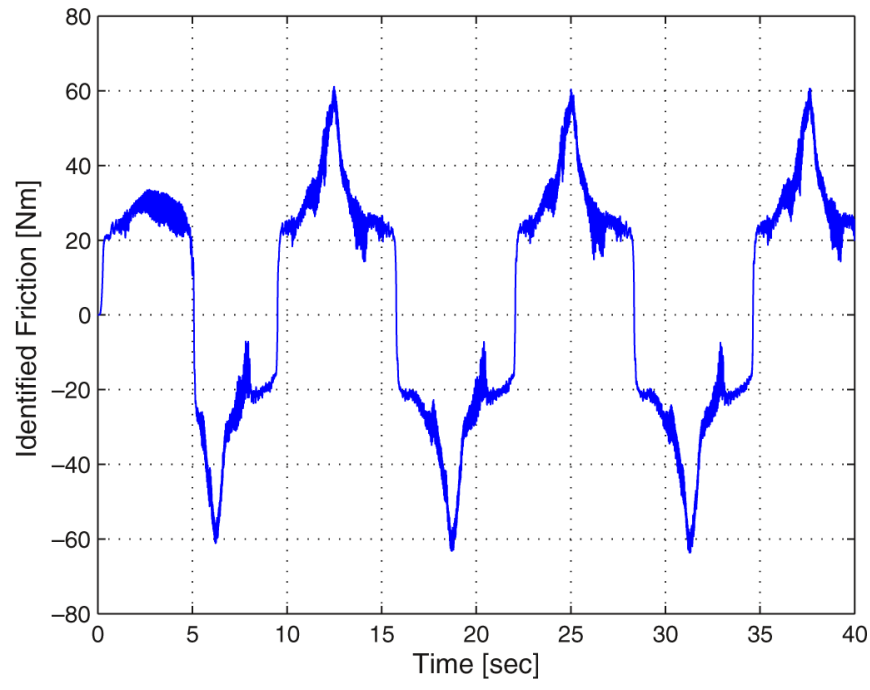

Fig. 3. Identified friction from the adaptive term in the proposed controller.

\section{Experiment 3}

The net external friction induced on the system as a result of external load applied to the circular disk by the linear thruster was identified. The friction in the testbed under no-load conditions was identified as in Experiment 1 using the control gains of Experiment 2. This identified friction term was subtracted from the identified friction terms obtained from Experiment 2. The friction between the circular disk and Nylon block can be seen in Fig. 4 .

\section{Experiment 4}

The experimentally identified friction torque using the adaptive term in (11) was compared with the friction torque model in (2). The matching of the friction torque with the experimental data is plotted in Fig. 5.

\section{DISCUSSION}

Experiment 1 illustrates an approximate factor of 60 improvement in the RMS tracking error over a PD controller, and a factor of approximately 4 over a typical exact model knowledge controller with 


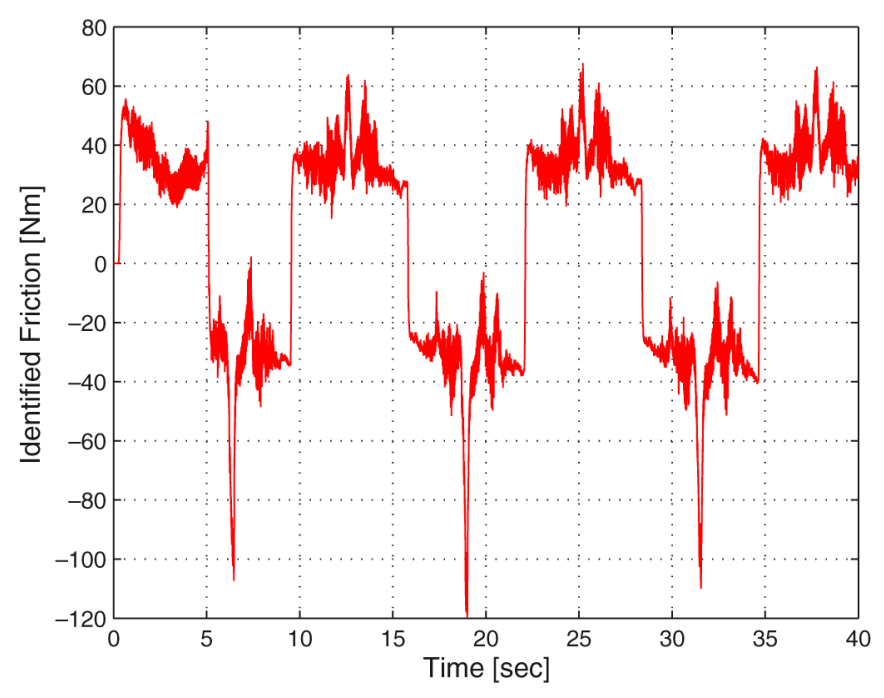

Fig. 4. Net external friction induced. The net friction was calculated by subracting the identified friction term in Experiment 1 from the identified friction term in Experiment 2.

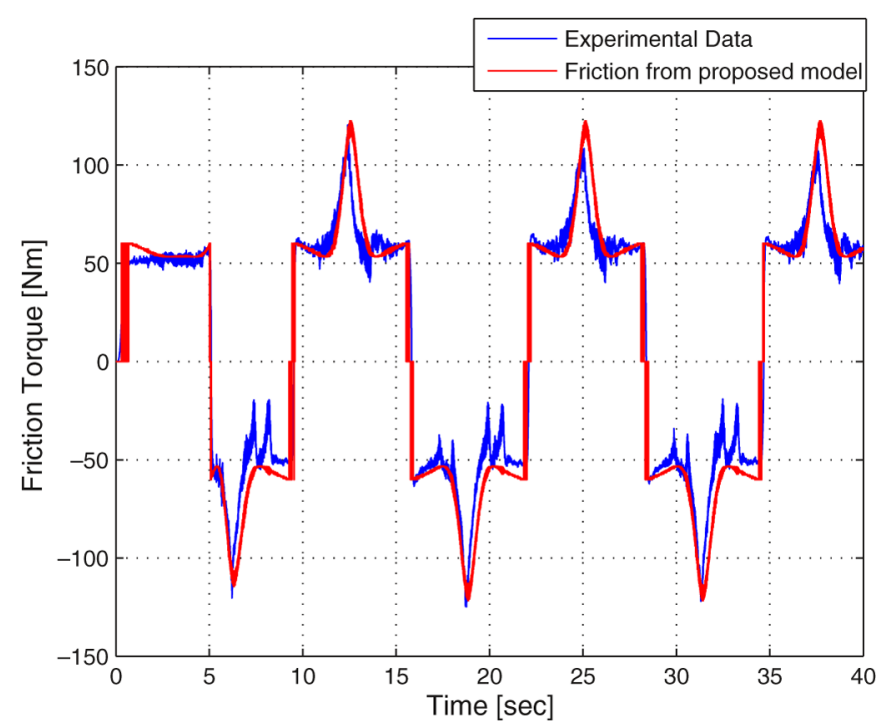

Fig. 5. The friction torque calculated from the model in (2) approximates the experimentally identified friction torque in (11).

static and viscous friction feedforward terms (see Figs. 1 and 2); Experiment 2 illustrates an approximate factor of 98 improvement in the RMS tracking error over the PD controller, and a factor of approximately 6 over the exact model knowledge controller. In Tables I and II, the RMS error from the proposed controller is approximately two orders of magnitude better than the PD controller and approximately one order of magnitude better than the model-based controller. This performance improvement was obtained while using similar or lower input torque.

The performance improvement is based on the fact that the proposed controller contains a feedforward term that identifies the friction as a general time-varying disturbance. To develop the friction identification term, the friction model is required to be continuously differentiable. Experiments 1-4 illustrate the identified friction torque. Specifically in Experiment 4, the parameters of the nonlinear parameterizable continuously differentiable friction model proposed in [24] were varied to
TABLE I

COMPARISON OF TRACKING RESULTS WHEN NO EXTERNAL LOAD WAS APPLIED TO THE CIRCULAR DISK

\begin{tabular}{|c|c|c|c|}
\hline & $\begin{array}{c}\text { PD } \\
\text { controller }\end{array}$ & $\begin{array}{c}\text { Model-based } \\
\text { controller }\end{array}$ & $\begin{array}{c}\text { Proposed } \\
\text { controller }\end{array}$ \\
\hline RMS error & 0.74 & 0.051 & 0.012 \\
\hline RMS torque & 33.77 & 37.28 & 32.11 \\
\hline
\end{tabular}

TABLE II

COMPARISON OF TRACKING RESULTS WHEN AN EXTERNAL LOAD WAS APPLIED TO THE CIRCULAR DisK

\begin{tabular}{|c|c|c|c|}
\hline & $\begin{array}{c}\text { PD } \\
\text { controller }\end{array}$ & $\begin{array}{c}\text { Model-based } \\
\text { controller }\end{array}$ & $\begin{array}{c}\text { Proposed } \\
\text { controller }\end{array}$ \\
\hline RMS error & 1.84 & 0.12 & 0.019 \\
\hline RMS torque & 83.38 & 103.87 & 73.96 \\
\hline
\end{tabular}

match the experimentally obtained friction torque. Fig. 5 shows that the proposed friction model in (2) approximates the experimental friction torque. However, since friction is anisotropic in nature, the magnitude of friction in experimental data is not symmetrical about the horizontal axis whereas the friction model in (2) approximates it as symmetric. Hence, future work can focus on mathematically distorting the model proposed in (2) by addition of more terms to make it asymmetric or making the unknown coefficients time-varying in order to capture more friction effects.

\section{CONCLUSION}

In this paper, semi-global asymptotic tracking is proven in the presence of a proposed continuously differentiable friction model that contains uncertain nonlinear parameterizable terms. To achieve the tracking result, an integral feedback compensation term is used to identify the system friction effects. A Lyapunov-based stability analysis is provided to conclude the tracking and friction identification results. Experimental results show two orders of magnitude improvement in tracking control over a proportional derivative (PD) controller, and a one order of magnitude improvement over the model-based controller. Experimental results are also used to illustrate that the experimentally identified friction can be approximated by the model in [24].

\section{REFERENCES}

[1] B. Armstrong-Helouvry, P. Dupont, and C. Canudas de Wit, "A survey of models, analysis tools and compensation methods for the control of machines with friction," Automatica, vol. 30, no. 7, pp. 1083-1138, 1994.

[2] N. Barahanov and R. Ortega, "Necessary and sufficient conditions for passivity of the LuGre friction model," IEEE Trans. Automat. Control, vol. 45 , no. 4 , pp. $830-832$, Apr. 2000.

[3] C. Canudas de Wit, "Robust control for servo-mechanisms under inexact friction compensation," Automatica, vol. 29, no. 3, pp. 757-761, Aug. 1993.

[4] C. Canudas de Wit and P. Lichinsky, "Adaptive friction compensation with dynamic friction model," in Proc. IFAC World Congress, San Francisco, CA, 1996, pp. 197-202.

[5] C. Canudas de Wit and P. Lichinsky, "Adaptive friction compensation with partially known dynamic friction model," Int. J. Adapt. Contr. Signal Processing, vol. 11, pp. 65-80, 1997.

[6] C. Canudas de Wit and R. Kelly, "Passivity-based control design for robots with dynamic friction model," in Proc. IASTED Int. Conf. on Robotics Manuf., Cancun, Mexico, 1997, pp. 84-87.

[7] C. Canudas de Wit, H. Olsson, K. J. Astrom, and P. Lischinsky, "A new model for control of systems with friction," IEEE Trans. Automat. Control, vol. 40, no. 3, pp. 419-425, Mar. 1995.

[8] Y.-Y. Chen, P.-Y. Huang, and J.-Y. Yen, "Frequency-domain identification algorithms for servo systems with friction," IEEE Trans. Contr. Syst. Tech., vol. 10, no. 5, pp. 654-665, Sep. 2002. 
[9] S.-I. Cho and I.-J. Ha, "A learning approach to tracking in mechanical systems with friction," IEEE Trans. Automat. Control, vol. 45, no. 1, pp. 111-116, Jan. 2000.

[10] D. M. Dawson, J. Hu, and T. C. Burg, Nonlinear Control of Electric Machinery. New York: Marcel Dekker, Inc., 1998.

[11] M. R. Elhami and D. J. Brookfield, "Sequential identification of coulomb and viscous friction in robot drives," Automatica, vol. 33, no. 3, pp. 393-401, 1997.

[12] M. Feemster, D. M. Dawson, A. Behal, and W. E. Dixon, "Tracking control in the presence of nonlinear dynamic frictional effects: Robot extension," Asian J. Contr., vol. 1, no. 3, pp. 153-168, 1999.

[13] B. Friedland and Y. J. Park, "On adaptive friction compensation," IEEE Trans. Automat. Control, vol. 45, no. 4, pp. 1609-1612, Apr. 2000.

[14] R. H. A. Hensen et al., "Grey-box modeling of friction: An experimental case-study," Europ. J. Contr., vol. 6, no. 3, pp. 258-267, 2000.

[15] R. H. A. Hensen, M. J. G. van de Molengraft, and M. Steinbuch, "Frequency domain identification of dynamic friction model parameters," IEEE Trans. Contr. Syst. Technol., vol. 10, no. 2, pp. 191-196, Mar. 2002.

[16] R. M. Hirschorn and G. Miller, "Control of nonlinear systems with friction," IEEE Trans. Contr. Syst. Technol., vol. 7, no. 5, pp. 588-595, Sep. 1999.

[17] S. Jain, F. Khorrami, N. Ahmad, and S. Sankaranarayanan, "Friction compensation for drives with and without transmission compliance," in Proc. IEEE Amer. Contr. Conf., Albuquerque, NM, 1997, pp. 2925-2929.

[18] H. K. Khalil, Nonlinear Systems, 3rd ed. Upper Saddle River, NJ: Prentice-Hall, 2002.

[19] S.-J. Kim and I.-J. Ha, "A frequency-domain approach to identification of mechanical systems with friction," IEEE Trans. Automat. Control, vol. 46, no. 6, pp. 888-893, Jun. 2001.

[20] S.-J. Kim, S.-Y. Kim, and I.-J. Ha, "An efficient identification method for friction in single-DOF motion control systems," IEEE Trans. Contr. Syst. Technol., vol. 12, no. 4, pp. 555-563, Jul. 2004.

[21] V. Lampaert, J. Swevers, and F. Al-Bender, "Modification of the Leuven integrated friction model structure," IEEE Trans. Automat. Control, vol. 47, no. 4, pp. 683-687, April 2002.

[22] T.-L. Liao and T.-I. Chien, "An exponentially stable adaptive friction compensator," IEEE Trans. Automat. Control, vol. 45, no. 5, pp. 977-980, May 2000.

[23] C. Makkar, W. E. Dixon, W. G. Sawyer, and G. Hu, "Lyapunov-based tracking control in the presence of uncertain nonlinear parameterizable friction," in Proc. IEEE Amer. Contr. Conf., Portland, OR, 2005, pp. 1975-1980.

[24] C. Makkar, W. E. Dixon, W. G. Sawyer, and G. Hu, "A new continuously differentiable friction model for control systems design," in Proc. IEEE/ASME Int. Conf. Adv. Intell. Mechatron., Monterey, CA, 2005, pp. 600-605.

[25] C. Makkar, "Nonlinear Modeling, Identification, and Compensation for Frictional Disturbances," Master thesis, University of Florida, , 2006.

[26] M. Loffler, N. Costescu, and D. Dawson, "Qmotor 3.0 and the Qmotor robotic toolkit-An advanced PC-based real-time control platform," IEEE Contr. Syst. Mag., vol. 22, no. 3, pp. 12-26, Jun. 2002.

[27] M. M. Polycarpou and P. A. Ioannou, "On the existence and uniqueness of solutions in adaptive control systems," IEEE Trans. Automat. Control, vol. 38, pp. 474-479, 1993.

[28] Z. Qu, Robust Control of Nonlinear Uncertain Systems. New York: Wiley, 1998.

[29] S. Sivakumar and F. Khorrami, "Friction compensation via variable structure control: Regulation and low velocity tracking," in Proc. IEEE Conf. Contr. Appl., Hartford, CT, 1997, pp. 645-650.

[30] J. Swevers, F. Al-Bender, C. G. Ganseman, and T. Projogo, "An integrated friction model structure with improved pre-sliding behavior for accurate friction compensation," IEEE Trans. Automat. Control, vol. 45, no. 4, pp. 675-686, Apr. 2000.

[31] P. Tomei, "Robust adaptive control of robots with arbitrary transient performance and disturbance attenuation," IEEE Trans. Automat. Control, vol. 44, pp. 654-658, 1999.

[32] P. Tomei, "Robust adaptive friction compensation for tracking control of robot manipulators," IEEE Trans. Automat. Control, vol. 45, no. 11, pp. 2164-2169, Nov. 2000.

[33] P. Vedagarbda, D. M. Dawson, and M. Feemster, "Tracking control of mechanical systems in the presence of nonlinear dynamic friction effects," IEEE Trans. Contr. Syst. Technol., vol. 7, pp. 446-456, Jul. 1999.
[34] B. Xian, D. M. Dawson, M. S. de Queiroz, and J. Chen, "A continuous asymptotic tracking control strategy for uncertain multi-input nonlinear systems," IEEE Trans. Automat. Control, vol. 49, no. 7, p. 1206, Jul. 2004, 1206.

[35] T. Zhang and M. Guay, "Comments on 'an exponentially stable adaptive friction compensator', 'IEEE Trans. Automat. Control, vol. 46, no. 11, pp. 1844-1845, Nov. 2001.

\section{On the Stabilization of Linear Systems Under Assigned I/O Quantization}

Bruno Picasso and Antonio Bicchi

\begin{abstract}
This paper is concerned with the stabilization of discrete-time linear systems with quantization of the input and output spaces, i.e., when available values of inputs and outputs are discrete. Unlike most of the existing literature, we assume that how the input and output spaces are quantized is a datum of the problem, rather than a degree of freedom in design. Our focus is hence on the existence and synthesis of symbolic feedback controllers, mapping output words into the input alphabet, to steer a quantized I/O system to within small invariant neighborhoods of the equilibrium starting from large attraction basins. We provide a detailed analysis of the practical stabilizability of systems in terms of the size of hypercubes bounding the initial conditions, the state transient, and the steady-state evolution. We also provide an explicit construction of a practically stabilizing controller for the quantized I/O case.
\end{abstract}

Index Terms-Controlled invariance, dynamic output feedback, practical stability, quantized systems.

\section{INTRODUCTION}

Quantization is a peculiar characteristic of many systems, which can be caused by analog-to-digital and digital-to-analog conversion, binary or digital sensors and actuators, etc. In other cases, it is necessary to introduce quantization of signals in order to reduce the information complexity of some sensors (such as, e.g., video cameras) by encoding it in a proper symbolic alphabet. Since [4], quantized control systems have been attracting increasing attention of the control community. Most recently, interest on quantization has been stimulated by the growing number of applications involving "networked" control systems, i.e., systems interconnected through communication channels of limited capacity [1], [9], [10], [14], [15].

This paper deals with the control of the dynamical system

$$
\left\{\begin{array}{l}
x(t+1)=A x(t)+b u(t) \\
y(t)=q(x(t)) \\
x \in \mathbb{R}^{n}, \quad u \in \mathcal{U} \subset \mathbb{R}, \quad y \in \mathcal{Y}, \quad t \in \mathbb{N}
\end{array}\right.
$$

Manuscript received September 9, 2004; revised February 13, 2006 and January 17, 2007. Recommended by Associate Editor J. Hespanha. This work was supported by the European Commission under IST-2004-004536 (IP) "RUNES" and IST-2004-511368 (NoE) "HYCON".

B. Picasso is with Scuola Normale Superiore, Pisa, Italy and Centro Interdipartimentale di Ricerca E. Piaggio, Università di Pisa, Pisa, Italy (e-mail: picasso.bruno@gmail.com).

A. Bicchi is with Centro Interdipartimentale di Ricerca E. Piaggio, Università di Pisa, Pisa Italy (e-mail: bicchi@ing.unipi.it).

Digital Object Identifier 10.1109/TAC.2007.904283 